\title{
Maltese Front Variability from Satellite Observations Based on Automated Detection
}

\author{
Matthew Lybanon
}

\begin{abstract}
Maltese Front location statistics are obtained from multichannel sea-surface temperature (MCSST) images, derived from Advanced Very High Resolution Radiometer (AVHRR) observations of the Mediterranean Sea during March 3 through September 27, 1993. The statistics are based on semiautomated determinations of the Front's sea-surface temperature surface expression. Expert analyses from the Naval Oceanographic Office give an accuracy check. Expert techniques are largely manual, labor-intensive, subjective, and skill-dependent; therefore, automation could be beneficial. A mathematical morphologybased method successfully delineates the Front. It finds the temperature gradients most likely to be the Front's and presents the corresponding segmentations to the operator. The method was developed to find stars in astronomical images, and has successfully analyzed solar magnetograms and satellite Gulf Stream images. Rings, fronts, and sunspots are not star-like, but simple preprocessing adapts the technique to these problem domains. This work constitutes another successful application. The success in "moving" the technique is encouraging. The Maltese Front's thermal gradients are 5-10 times weaker than the Gulf Stream North Wall's, yet the method produces useful results, and it may work in other regions.
\end{abstract}

\section{INTRODUCTION}

$\mathbf{H}$ UMAN expert analysts derive useful oceanographic information from satellite observations, but the techniques are largely manual, labor-intensive, and are both subjective and highly dependent on the interpreter's skill. An automated or semiautomated system could provide a useful alternative. A method that uses the opening and closing operations of mathematical morphology [1], [2] shows success in delineating the Maltese Front in satellite images comparable to prior Gulf Stream results [3], [4]. The method finds temperature gradients most likely to be the Front's and presents the corresponding image segmentations to the operator, who may overrule those choices. The results may be input to further processing, e.g., feature labeling, edge linking, etc. [5].

The Maltese Front is the boundary between two water masses evidently formed by surface water from the western Mediterranean and by the Ionian Sea. During part of the year, it is oriented predominantly north-south and is located along the continental slope east of Malta from Sicily's southern tip to about $35^{\circ} 30^{\prime} \mathrm{N}$. At other times, its orientation is more eastwest, and it is located between Sicily and Malta. The region has comparatively shallow bathymetry, generally less than 200 $\mathrm{m}$. The Front's surface thermal expression is also much weaker than the Gulf Stream's; the magnitude of the gradient is about

Manuscript received November 6, 1995; revised April 2, 1996.

The author is with the Naval Research Laboratory, Stennis Space Center, MS 39529 USA.

Publisher Item Identifier S 0196-2892(96)06827-1. $1.5^{\circ} \mathrm{C}$ or less, and is often less than $1^{\circ} \mathrm{C}$. So, it is noteworthy that a technique that works well in the Gulf Stream area also succeeds in this very different regime.

The study reported in this paper demonstrates the method's utility by example, by using it to extract the information for an oceanographic analysis of the Maltese Front's position variability. Not only does this accomplish the objective, but examination of the method's results provides insight into some practical matters connected with its use.

\section{THE METHOD}

\section{A. Mathematical Morphology}

Extensive discussions and reviews of mathematical morphology may be found in [1], [2], [6]. Mathematical morphology is an approach to image processing based on shape which, if used appropriately, can simplify image data without losing essential shape characteristics. Morphologic operators work with two images: the data to be analyzed and a structuring element (usually smaller), analogous to a convolution kernel. The structuring element is a geometric pattern of pixels that may be thought of as a probe which finds specific shapes in the (other) image.

Two basic morphological operations are dilation and erosion. Dilation combines two images by vector addition of set elements. The dilation operator fills "holes" in the image equal to or smaller than a particular structuring element. Erosion, the morphological opposite of dilation, combines two images by vector subtraction of set elements. The erosion operator eliminates islands smaller than a particular structuring element. (These are qualitative statements, and refer primarily to the type of structuring element used in this paper's method.) Opening is erosion followed by dilation of the eroded image. Closing is dilation followed by erosion of the dilated image. A binary image that has been both opened and closed cannot be transformed further by either operation [1].

The gray-level image operations can be visualized by regarding the image and the structuring element as (intensity) surfaces. If the entire structuring element fits under or at the image surface, the eroded image pixel corresponding to the structuring element's origin is given the minimum intensity of all image pixels "spanned" by the structuring element. If any structuring element pixel fits under or at the image surface, the dilated image pixel is given the maximum intensity of all image pixels spanned by the structuring element. The structuring element's origin is often its center pixel, but may be any pixel, even one outside the structuring element. If the 
structuring element pixels all have equal value, that "height" creates an intensity threshold for the image.

\section{B. The Algorithm}

The method used in the present study uses a square (usually a $3 \times 3$ gives the smoothest edges), constant-value structuring element. The essence of the procedure is to open the image and then close the opened image at a particular threshold. The output of this step is the input to the next step, opening and closing at a higher threshold. The process is repeated until a stable division into objects and background is found.

Choosing a threshold is equivalent to defining (tentatively) an object boundary's intensity; incrementing it is equivalent to (tentatively) redefining that level. The increment is related to an estimate of the intensity difference at edges of interest. When the threshold is twice incremented near an edge intensity, only a few pixels change from object to background or vice versa on the second iteration.

The basic open-close algorithm is described in more detail in [7], which describes its use to find stars in astronomical images. The changes necessary to adapt the method to one new problem domain, finding ocean features in satellite infrared images, are described in [3] and [4]. Note that the type of processing is what is called an "alternating sequential filter" in [8].

\section{Adaptation to the Problem}

The method is not problem-specific; it has been applied successfully to find stars in astronomical images, ocean features in infrared (IR) images of the Gulf Stream region, sunspots in solar magnetograms, and in the present application [9]. Only some preprocessing and other minor changes are necessary to adapt this robust technique to different problems.

The software permits the replacement of foreground values with those pixels' original image intensities, instead of the values that result from a strict application of the mathematical morphology definitions. It is sometimes desirable to retain the original values in this way. A related option is to choose the foreground to be made up of pixels below the threshold the structuring element defines, rather than above it. More than one step can be performed (e.g., to find pixels between two thresholds). These different options were used in previous applications of this technique.

The Maltese Front application starts with multichannel seasurface-temperature (MCSST) images of the Mediterranean Sea. An algorithm that combines channels of the Advanced Very High Resolution Radiometer (AVHRR) on NOAA polarorbiting satellites produces a numerical sea-surface temperature (SST) estimate with the sensor's $0.1{ }^{\circ} \mathrm{C}$ resolution; brightness increases with temperature. The water on one side of the Maltese Front is typically the coldest "object" (along with clouds) in the image, so a single-step process that eliminates colder objects can find it. The stability (convergence) criterion is a minimum in the number of pixels changing classification. A method for choosing a reasonable initial threshold that depends on the time of year, based on study of

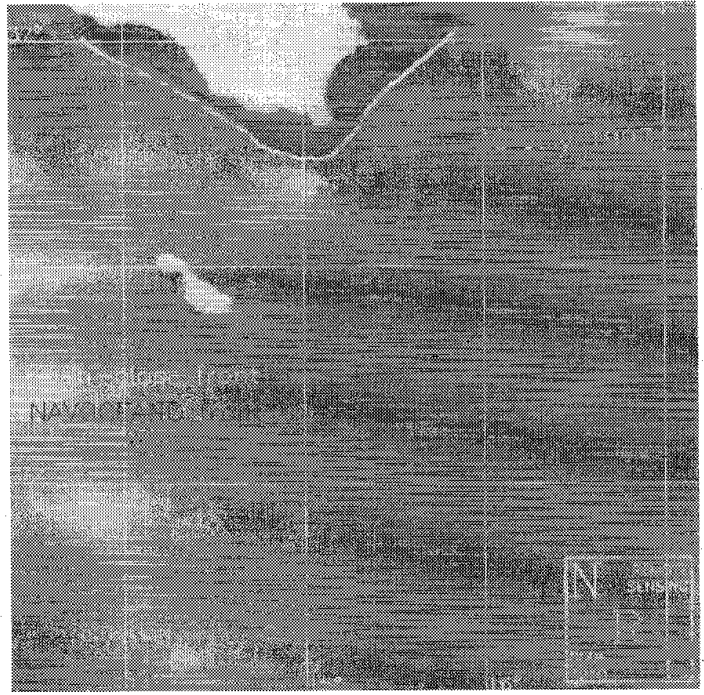

Fig. 1. July 30, 1993 SST image from NOAA-11 AVHRR data, with the results of the open-close (white line) and NAVOCEANO (black line) analyses superimposed. The southern tip of Sicily is the white shape just above the lines. Malta is the smaller white shape in the center left part of the image. The Maltese Front appears to be the temperature step close to the lines.

a large set of images, reduces the number of iterations needed to produce results.

The open-close algorithm, like other mathematical morphology applications, is not information-preserving. The "philosophy" is to suppress irrelevant information to obtain an image that is better suited for the purpose, which, in the present case, is location of the Maltese Front. The details of temperature variation on one side (the cold side) of the front are irrelevant. The resulting image, after processing by the algorithm, shows a distinct, continuous (except where clouds obscure the original image) boundary that shows the method's estimate of the Maltese Front's location.

\section{PROCESSING}

\section{A. Front Detection}

Fifty-four $256 \times 256$ subimages were extracted from larger 16-b SST images of a portion of the Mediterranean Sea, acquired during March 3 to September 27, 1993. Forty-nine were usable for this study. Each subimage covers the same area, $34.04^{\circ}$ to $37.16^{\circ}$ north latitude and $13.36^{\circ}$ to $17.18^{\circ}$ east longitude. The subimages were further converted to $8-\mathrm{b}$ data while maintaining the sensor's full $0.1^{\circ} \mathrm{C}$ temperature resolution by extracting the range $7.5^{\circ} \mathrm{C}$ through $33.0^{\circ} \mathrm{C}$.

Figs. 1 and 2 illustrate the open-close processing. Fig. 1 shows the SST image for July 30, 1993 and Fig. 2 shows the result of applying the open-close method. The white shape at the top left of each image is Sicily's southern tip, and the smaller white shape is Malta. Superimposed on Fig. 1 is the front derived from the open-close results and, for comparison, the front from the Naval Oceanographic Office's (NAVOCEANO) operational analysis. This analysis is the result of expert human interpretation of satellite imagery (in many cases, the same images used in the present study) 


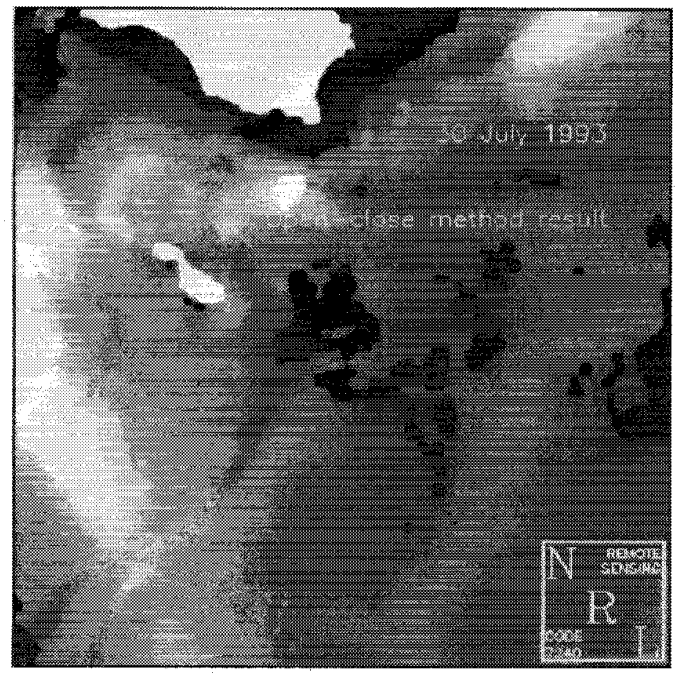

Fig. 2. Result of applying the open-close technique to the image of Fig. 1. The Maltese Front is taken to be the boundary between the black and lighter gray shades near the top of the image. It is close to the apparent location of the Front in Fig. 1.

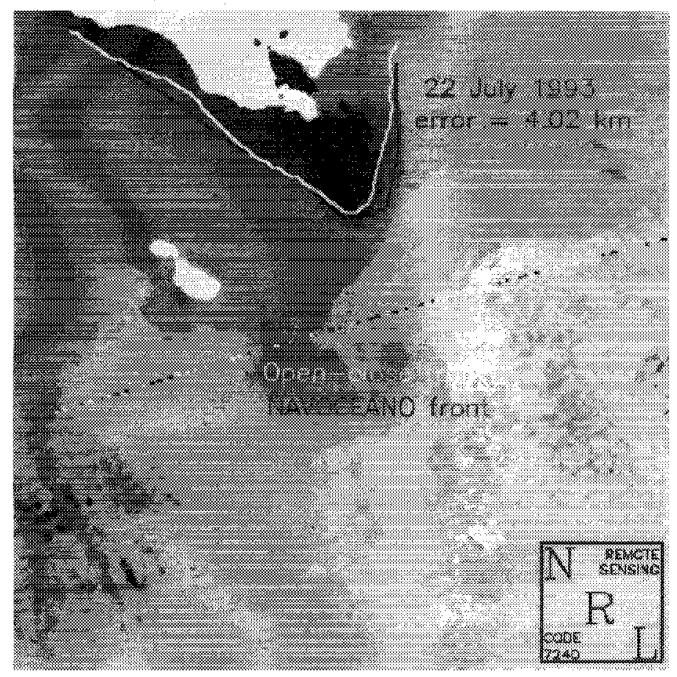

Fig. 3. July 22, 1993 SST image from NOAA-11 AVHRR data, showing the open-close and NAVOCEANO fronts as in Fig. 1, and the error in the former's position relative to the latter. The error would be smaller if more points were used to define the NAVOCEANO front.

and, when available, in situ measurements. The black area surrounding Sicily's southern tip in Fig. 2 is the background found by the open-close method, and the boundary between it and foreground pixels is an estimate of front location.

Finding the location of a front in a SST image requires knowing the highest temperature of the pixels on the cold side of the front ("frontmax"). The system then smooths the temperature values in the image and zeroes pixels with temperatures no greater than this value. The aim of the system (or human analyst) is to use information contained in the image to reduce the number of choices for frontmax from 256 to one or a few, among which is the correct value.

The first processing step is calculation of image statistics. Study of the statistics of all images in the data set led to a
TABLE I

\begin{tabular}{|c|c|c|c|}
\hline Mean & 10.05 & $\begin{array}{l}\text { Standard } \\
\text { deviation }\end{array}$ & 5.40 \\
\hline Minimum & 4.02 & Maximum & 28.64 \\
\hline \multicolumn{4}{|c|}{ TABLE II } \\
\hline Mean & 25.68 & $\begin{array}{l}\text { Standard } \\
\text { deviationm }\end{array}$ & 21.39 \\
\hline Minimum & 5.05 & Maximum & 141.70 \\
\hline
\end{tabular}

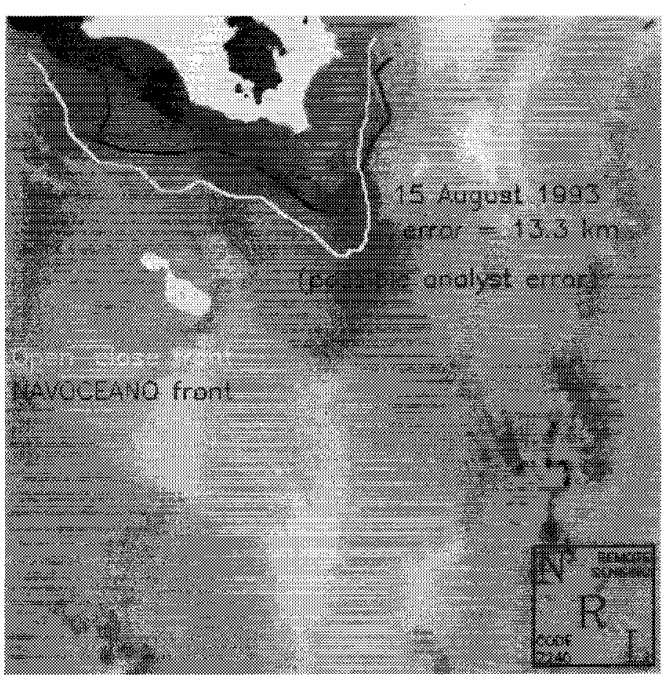

Fig. 4. August 15 SST image from NOAA-11 AVHRR data, showing the open-close and NAVOCEANO fronts, and the error in the former's position relative to the latter. The open-close front may have been located incorrectly, as Fig. 5 illustrates.

procedure to specify an initial threshold based on the date and quality ("image quality" is one of the statistics) of the image that yields frontmax estimates in a small number of iterations. The program opens and closes the image, and the number of pixels that change classification from background to object or vice versa is found for each iteration.

The program finds ranges containing minima (in the number of pixels changing category), and presents the analyst with up to four segmented images (with pixels whose smoothed temperatures less than the candidate frontmax are set to zero), ranked in order of the likelihood that each contains the correct frontmax. The user may choose to accept (and save) one of them as accurately delineating the front, to explore other possible frontmax values in a range, or to select an arbitrary frontmax.

\section{B. Statistical Analysis}

Validation of the open-close method and calculation of frontal statistics from its results are the two main parts of the analysis. Validation consists of comparing the results with corresponding NAVOCEANO daily analyses, which are taken to be "ground truth." Individual open-close results are compared with a mean Maltese Front to produce position variability statistics. 


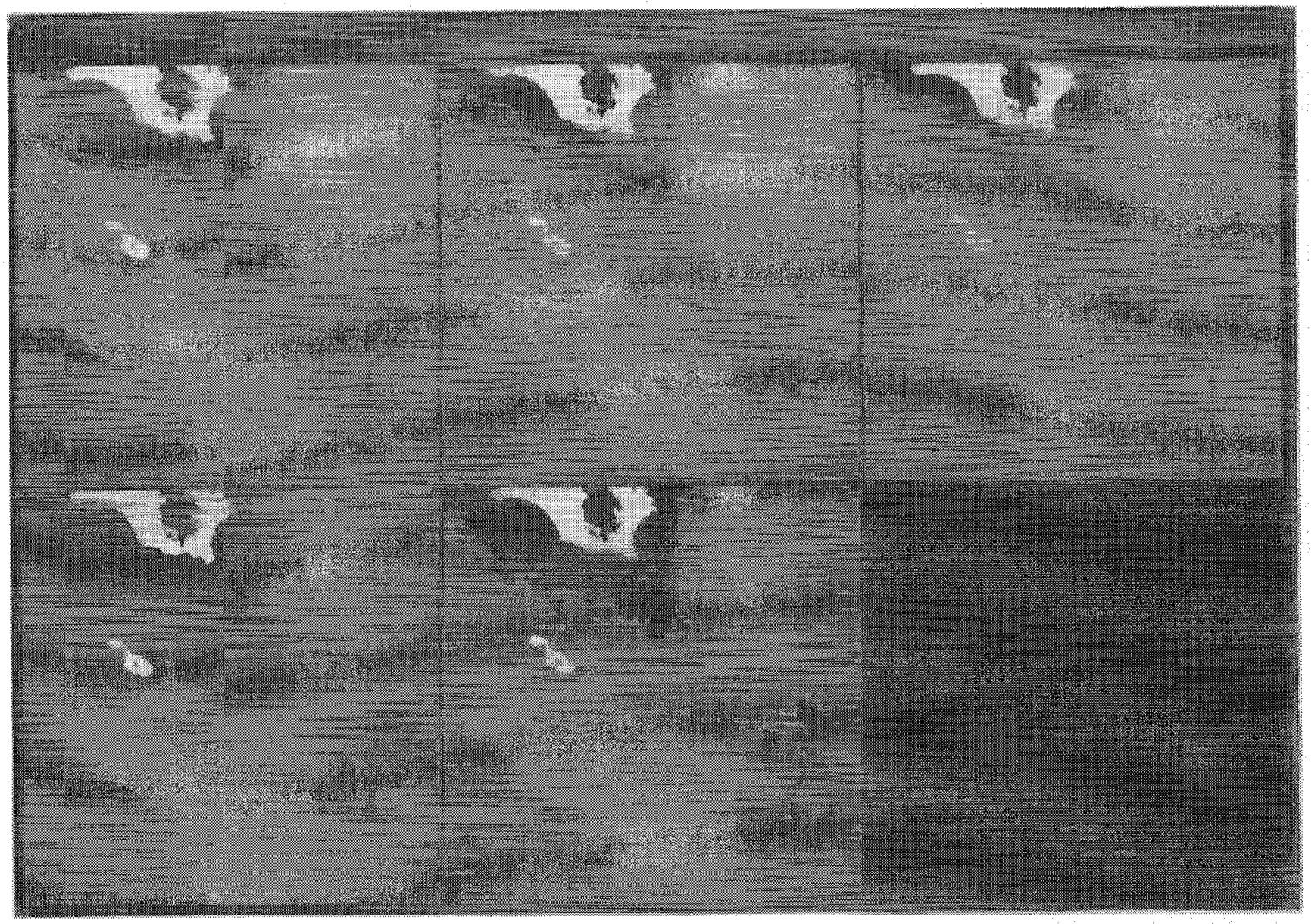

Fig. 5. The upper left panel shows the August 15 image (as in Fig. 4). The other two upper panels and the lower center panel show candidate segmentations initially presented to the analyst by the open-close software. The analyst chose the lower center segmentation, leading to the (possibly erroneous) open-close front shown in Fig. 4. The lower left panel is the result of further "probing" at the analyst's request.

This procedure requires concurrent open-close results and NAVOCEANO analyses. There are 24 such cases. Two fronts are compared over a specified longitude range by interpolating each to a standard grid. The error is estimated by calculating the area between them and dividing that area by the arclength of the "ground truth" frontal segment within that longitude range. The result is a mean error, expressed in kilometers. Statistics are calculated from individual errors.

The NAVOCEANO daily analyses served as ground truth and the open-close results were compared with them for validation. A mean Maltese Front calculated from the openclose results served as the standard for the position variability analysis. Comparison of the individual open-close results with the mean front provided deviations from the mean.

Tables I and II summarize the results. Table I presents the validation of the open-close method statistics of the deviations of open-close fronts from NAVOCEANO fronts. It lists mean and standard deviation, minimum, and maximum errors, all in kilometers. Table II presents the comparable statistics for the variation of the open-close fronts about the mean front found from them.

\section{DiscusSION OF RESUltS}

\section{A. Validation}

Examination of the individual results is instructive. Not only do the results give concrete meaning to the statistics, but in some cases, they illustrate features of the open-close technique in practice. All images are of the same area shown in Figs. 1 and 2. All dates are in 1993. Fig. 3 is the image for July 22, with the open-close and NAVOCEANO fronts superimposed, along with the open-close result's deviation from the NAVOCEANO front, expressed as an error (see above discussion). This case has the smallest error of any tested, although the July 30 case (Fig. 1) has only a slightly larger error, $4.08 \mathrm{~km}$ instead of $4.02 \mathrm{~km}$.

The NAVOCEANO analysis results are lists of points along what the analyst considers to be the front. The "NAVOCEANO analyses" used as ground truth in this study's calculations are broken-line approximations, obtained by connecting the points with straight lines. Connecting the points with cubic splines or some other smooth curves would result in somewhat different calculated errors-smaller in some cases.

The largest error found is for June 5 (not shown), $28.6 \mathrm{~km}$. For this case, the NAVOCEANO front is only $56.6 \mathrm{~km}$ long. In contrast, for July 22 , the mean error is calculated along a 235 $\mathrm{km}$ arclength. The open-close front for June 5 is comparatively far from the NAVOCEANO front in the region where they are both defined, yielding a large error. This result is a statistical quirk.

Fig. 4 shows the August 15 image with the same information superimposed as that in Fig. 3. Fig. 5, minus the lower-left frame, shows the candidate segmentations that the open-close program provided to the operator. The operator chose the one 


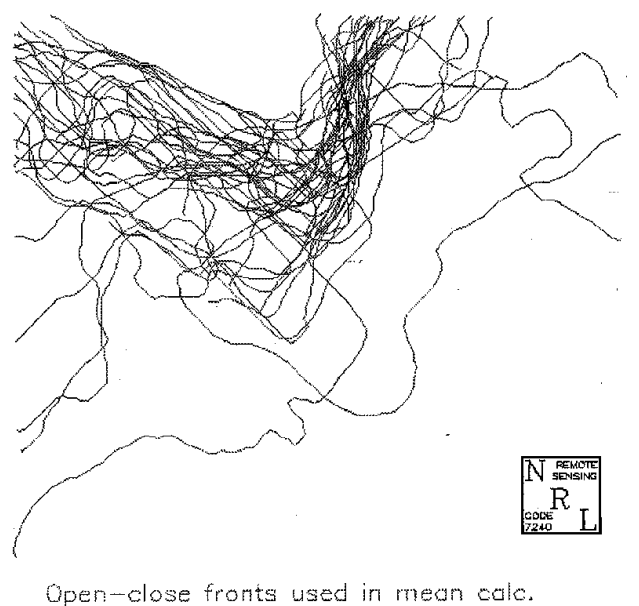

Fig. 6. The individual open-close fronts that went into the mean Maltese Front calculation.
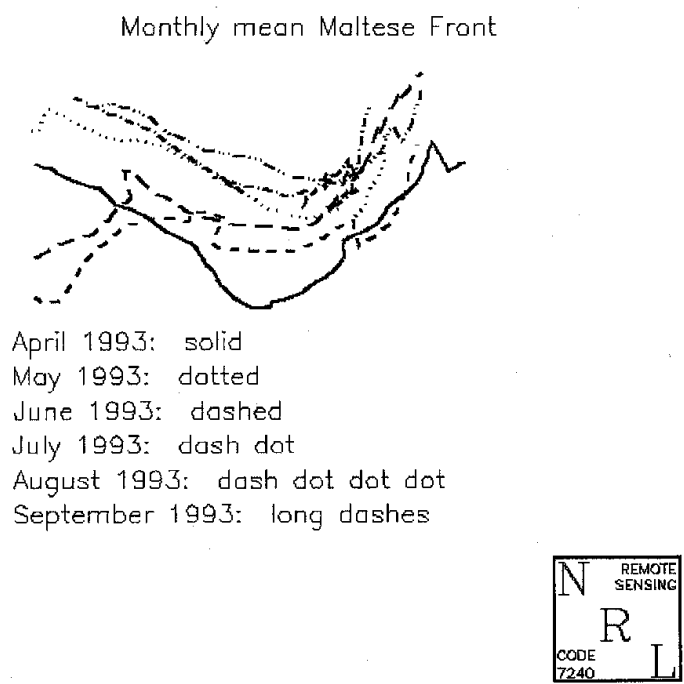

Fig. 7. Monthly mean Maltese Fronts, for April through September 1993 , as found by the open-close method. The "mean front" shown in subsequent figures is the mean of all the fronts shown in Fig. 6, not one of the monthly means.

in the lower row's center, leading to the open-close front shown in Fig. 4. A more experienced operator, referring back to the original image, would be likely to direct the program to explore other possible frontmax values. The lower-left frame in Fig. 5 is the result of such further exploration and would lead to an open-close front that is closer to the NAVOCEANO front. This case emphasizes that the open-close technique is a tool to assist the analyst. As is the case with most tools, skill increases with practice.

It is of interest to identify and analyze cases for which the open-close technique yields poor results. As the case illustrated in Figs. 4 and 5 shows, the quality of the results depends partly on the quality of the analyst's judgment in using the information the open-close algorithm presents. But if the initial candidate segmentations are frequently rejected by a careful analyst, the logic used to obtain them is suspect. The present

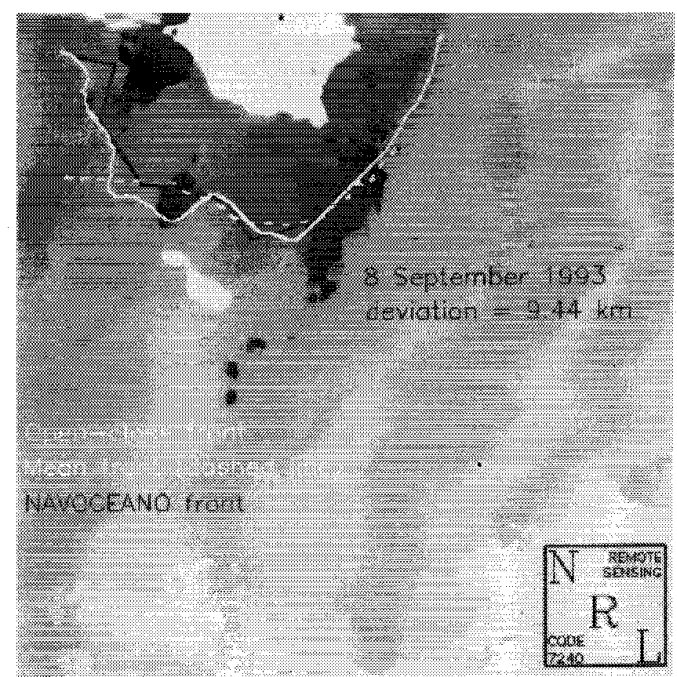

Fig. 8. September 8, 1993 SST image from NOAA-11 AVHRR data, showing the open-close (solid white line) and the NAVOCEANO (solid black line) fronts, the mean front (dashed white line), and the deviation of the open-close front from the mean front. The open-close front's position is well validated by the NAVOCEANO front, and is also close to the mean front.

data set is too small to investigate this question adequately, but use of the method in a quasi-operational setting over a period of time could be of value.

\section{B. Variability Analysis}

This is a comparatively small-scale study, covering a period of less than a full year. The primary purpose of performing this analysis is to illustrate the open-close method's utility in extracting oceanographic information from satellite observations. The extracted information is in a form convenient for further analysis. The form of the analysis would be similar for a larger study.

Each Maltese Front found by the open-close technique begins and ends at a different place. The procedure adopted to circumvent this difficulty was to "resample" each front to a common datum, extrapolating where necessary, with consecutive points equally spaced along the front. In this way, each front was given the same starting point, where it crosses $13^{\circ} \mathrm{E}$ longitude, and the same length, 121 points ( $5 \mathrm{~km}$ apart). The resampled fronts then went into a point-by-point mean calculation. The extrapolated points were given zero weight in the mean calculation; their function was merely to "locate" the real data. So points in the middle of the mean front had contributions from more individual fronts than points near the ends.

Fig. 6 shows the (original, not resampled) fronts that went into the mean calculation. The resulting mean had to be truncated somewhat because a few of the points at each end were not stable statistical means, coming from averaging too few individual fronts. The "usable" portion of the mean front was taken to be the part between $13.61^{\circ}$ and $15.59^{\circ} \mathrm{E}$ longitude.

Monthly means can be calculated in the same way. Although the means derived from this data set are not of high quality, 


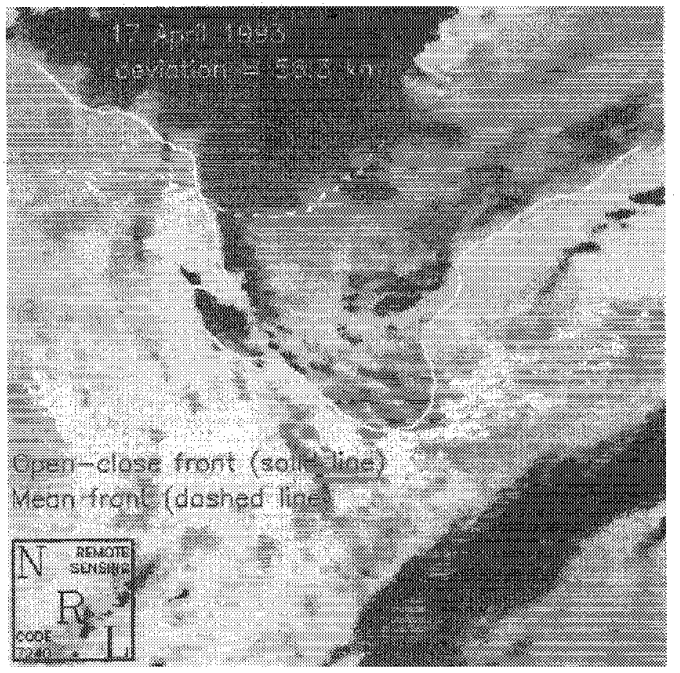

Fig. 9. April 17, 1993 SST image from NOAA-11 AVHRR data, showing the open-close front, the mean front, and the deviation of the former from the latter. There is no NAVOCEANO front for this day, but open-close analyses for April 7 and 21 are consistent with this open-close result (see Fig. 10). So, the large deviation from the mean front is probably genuine.

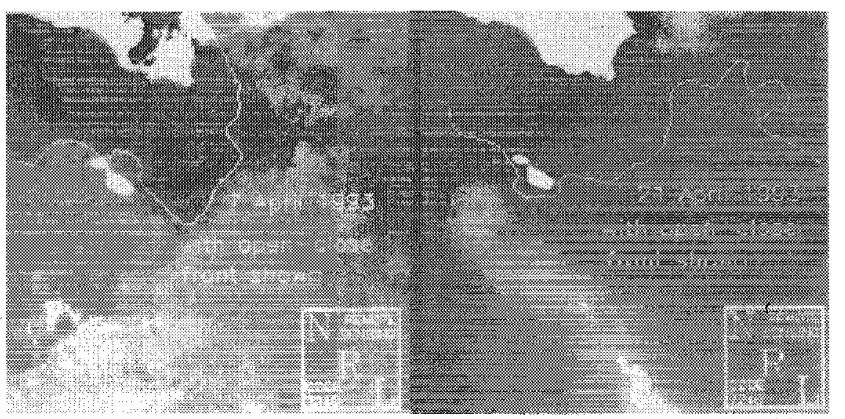

(a)

(b)

Fig. 10. (a) April 7, 1993 SST image from NOAA-11 data, with open-close front superimposed (left). (b) April 21, 1993 SST image from NOAA-11 data, with open-close front superimposed (right).These front positions are consistent with the open-close front's position in Fig. 9.

owing to the small size of the data set, information on monthly or seasonal position variability could be readily obtained using the method of this paper. Fig. 7 shows the monthly means so obtained. The monthly means are also truncated to "useful" lengths. Only the March mean is absent since it was obtained by averaging only (at most) two fronts.

Fig. 8 shows the September 8 case, whose open-close front agrees most closely with the mean front; the deviation is $9.44 \mathrm{~km}$. Superimposed on the image are the open-close, NAVOCEANO, and mean fronts. Comparison of the first two of these shows that the open-close front's agreement with the ground truth is also good (error $=4.35 \mathrm{~km}$ ). In contrast, the July 22 open-close front is much farther from the mean front, $29.7 \mathrm{~km}$. But reference to Fig. 3 shows that this case's openclose front agrees very well with the ground truth. It is the case with the best agreement. Therefore, it is likely that the substantial difference in deviations of these two cases from the mean front are reflections of actual variability in the Maltese Front's position over time, rather than an error.

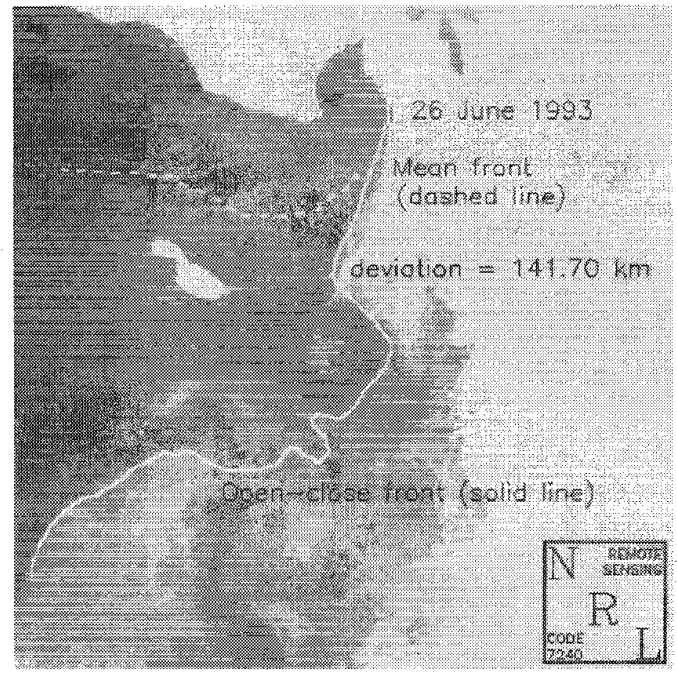

Fig. 11. June 26, 1993 SST image from NOAA-11 data, showing the open-close front, the mean front, and the deviation of the former from the latter. The large deviation is probably genuine (see Fig. 12).

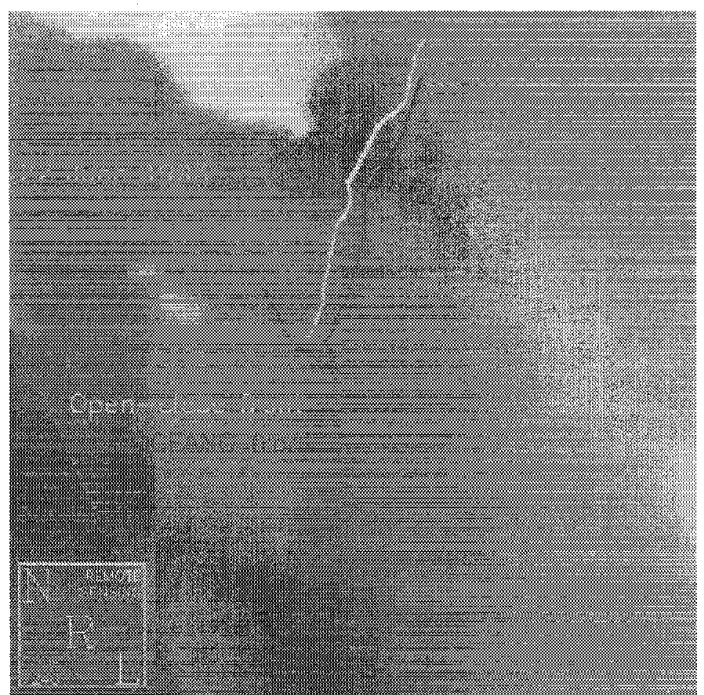

Fig. 12. June 22, 1993 SST image from NOAA-11 data, with the results of the open-close and NAVOCEANO analyses superimposed. Since the open-close front is consistent with that for June 26 where the former is visible, and the June 22 open-close result is closely validated by the NAVOCEANO analysis, the large deviation for June 26 is concluded to be due to actual variability in the front's position.

In contrast, for April 17 (Fig. 9) the open-close front's deviation from the mean front is $56.3 \mathrm{~km}$, more than twice the average deviation from the mean. There is no NAVOCEANO front for the date to validate the open-close result. However, the results for the closest dates before and after, April 7 and April 21 [Fig. 10(a) and (b)], are similar. This consistency supports the hypothesis that the result is correct, since the open-close method has been validated in general.

The June 26 open-close front's deviation from the mean Maltese Front is even greater, $141.70 \mathrm{~km}$ (Fig. 11). But the result for June 22, shown in Fig. 12, tends to confirm the open-close result. The open-close front for June 22 is in a 
position close to that found for June 26, and the June 22 result is well confirmed by the NAVOCEANO analysis for that date.

\section{CONCLUSION}

The open-close method produces good edges for use in tracking the motion of ocean features. The edges found are continuous unless they are obscured by cloud cover. The method facilitates image segmentation by removing much extraneous information from the input images. The Maltese Front locations found with the aid of this method show good correlation with the results of expert analysis. In some respects, they may be better since the resolution is higher (the human analyst selects a small number of points on what is considered to be the front boundary).

This paper presents summary statistics on Maltese Front variability. The monthly means give an idea how variability changes with time of year. This data set's small number of values in a single month limits the quality of the monthly means. This could be remedied with a larger data set which would increase the reliability of all the results. But the present work illustrates the method's potential utility in performing oceanographic analyses. Application of the open-close method to synthetic aperture radar observations of the same area is currently under way.

\section{REFERENCES}

[1] R. M. Haralick, S. R. Sternberg, and X. Zhuang, "Image analysis using mathematical morphology." IEEE Trans. Pattern Anal. Machine Intell., vol. 9, pp. 532-550, 1987.
[2] J. Serra, Image Analysis and Mathematical Morphology. New York: Academic, 1982, pp. 34-62.

[3] S. M. Lea and M. Lybanon, "Finding mesoscale ocean structures with mathematical morphology," Remote Sensing Environ., vol. 44, pp. 25-33, 1992.

[4] _ـ "Automated boundary delineation in infrared ocean images," IEEE Trans. Geosci. Remote Sensing, vol. 31, pp. 1256-1260, 1993.

[5] M. Lybanon, S. Peckinpaugh, R. Holyer, and V. Cambridge, "Integrated ocean understanding system," in Image Understanding in the '90s: Building Systems That Work. Bellingham, WA: SPIE 1406, 1990, pp. 180-189.

[6] S. R. Sternberg, "Grayscale morphology," Comput. Vision. Graphics, Image Process., vol. 35, no. 3, pp. 333-355, Sept. 1986.

[7] S. M. Lea and L. A. Kellar, "An algorithm to smooth and find objects in astronomical images," Astron. J., vol. 97, pp. 1238-1246, 1989.

[8] H. J. A. M. Heijmans, "Composing morphological filters," Amsterdam, The Netherlands, Rep. BS-R9504, CWI, 1995

[9] M. Lybanon, S. M. Lea, and S. M. Himes, "Segmentation of diverse image types using opening and closing," in Proc. 12th IAPR Int. Conf. Pattern Recognition, Jerusalem, Israel, Oct. 9-13, 1994, vol. 1, pp. 347-351.

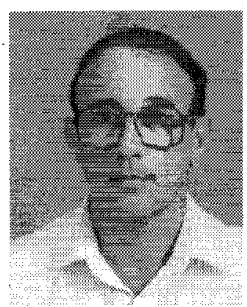

Matthew Lybanon received the B.S. and M.S. degrees in physics from the Georgia Institute of Technology, Atlanta, in 1960 and 1962, respectively.

$\mathrm{He}$ is a Principal Investigator in the Remote Sensing Applications Branch of the Naval Research Laboratory, Stennis Space Center, MS. His current research interests include genetic algorithms, image processing, mathematical morphology, expert systems, generalized nonlinear least squares methods, and applications of those topics to the extraction of information on ocean dynamics and shallow-water bathymetry from satellite observations. 